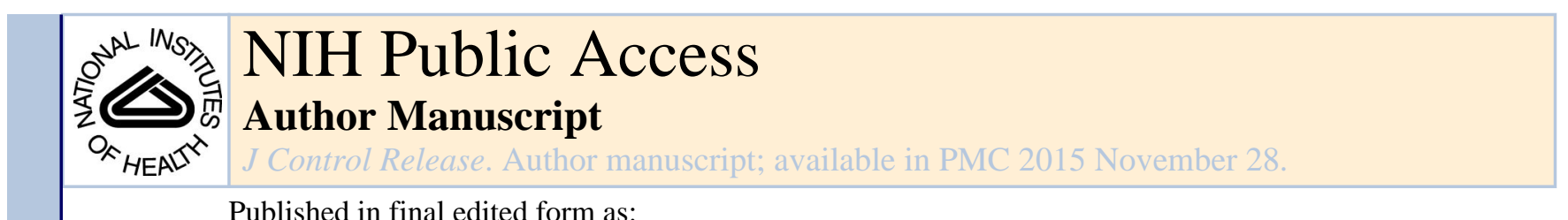

Published in final edited form as:

J Control Release. 2014 November 28; 194: 113-121. doi:10.1016/j.jconrel.2014.08.020.

\title{
ENHANCED GENE DELIVERY IN PORCINE VASCULATURE TISSUE FOLLOWING INCORPORATION OF ADENO- ASSOCIATED VIRUS NANOPARTICLES INTO POROUS SILICON MICROPARTICLES
}

\author{
Kellie I. McConnell ${ }^{1}$, Jessica Rhudy ${ }^{1}$, Kenji Yokoi ${ }^{1}$, Jianhua Gu ${ }^{1}$, Aaron Mack ${ }^{1}$, Junghae \\ Suh $^{2}$, Saverio La Francesca ${ }^{3}$, Jason Sakamoto ${ }^{1}$, and Rita E. Serda ${ }^{1,4,{ }^{*}}$ \\ ${ }^{1}$ Department of Nanomedicine, Houston Methodist Research Institute, Houston, Tx \\ ${ }^{2}$ Department of Bioengineering, Rice University, Houston, TX \\ ${ }^{3}$ Methodist DeBakey Heart and Vascular Center Methodist Hospital, Houston, TX \\ ${ }^{4}$ Department of Surgery, Baylor College of Medicine, Houston, TX
}

\begin{abstract}
There is an unmet clinical need to increase lung transplant successes, patient satisfaction and to improve mortality rates. We offer the development of a nanovector-based solution that will reduce the incidence of lung ischemic reperfusion injury (IRI) leading to graft organ failure through the successful ex vivo treatment of the lung prior to transplantation. The innovation is in the integrated application of our novel porous silicon ( $\mathrm{pSi}$ ) microparticles carrying adeno-associated virus (AAV) nanoparticles, and the use of our ex vivo lung perfusion/ventilation system for the modulation of pro-inflammatory cytokines initiated by ischemic pulmonary conditions prior to organ transplant that often lead to complications. Gene delivery of anti-inflammatory agents to combat the inflammatory cascade may be a promising approach to prevent IRI following lung transplantation. The rationale for the device is that the microparticle will deliver a large payload of virus to cells and serve to protect the AAV from immune recognition. The microparticlenanoparticle hybrid device was tested both in vitro on cell monolayers and ex vivo using either porcine venous tissue or a pig lung transplantation model, which recapitulates pulmonary IRI that occurs clinically post-transplantation. Remarkably, loading AAV vectors into pSi microparticles increases gene delivery to otherwise non-permissive endothelial cells.
\end{abstract}

\footnotetext{
(C) 2014 Elsevier B.V. All rights reserved

*To whom correspondence should be addressed: Rita E. Serda, Ph.D., The Methodist Hospital Research Institute, Department of NanoMedicine, 6670 Bertner Ave, R7-414, Houston, TX 77030, Phone: 713-204-2105, ritaserda@ gmail.com.

Publisher's Disclaimer: This is a PDF file of an unedited manuscript that has been accepted for publication. As a service to our customers we are providing this early version of the manuscript. The manuscript will undergo copyediting, typesetting, and review of the resulting proof before it is published in its final citable form. Please note that during the production process errors may be discovered which could affect the content, and all legal disclaimers that apply to the journal pertain.
} 


\section{Keywords}

mesoporous silicon; adeno-associated virus; inflammation; gene therapy; lung; endothelium; ex vivo perfusion

\section{Introduction}

Acute lung injury (ALI) and its most severe manifestation, acute respiratory distress syndrome (ARDS), is a clinical syndrome defined by acute hypoxemic respiratory failure and bilateral pulmonary infiltrates consistent with edema. In-patient mortality is $38.5 \%$ for ALI, and $41.1 \%$ for ARDS.[1] Common causes of ALI/ARDS are sepsis, trauma, multiple blood transfusion, aspiration, and injury from toxic inhalation [1-3]. At this time, low tidal volume ventilation remains the only intervention that affords mortality benefit. Therefore, clinical approaches based on interruption of pathways identified in the acute phase are of interest for the development of strategies that can significantly lower the current mortality rates. Typically, a patient"s intrapulmonary inflammatory response begins prior to the onset of clinically defined ALI and is most intense in the first 3 days after the onset of ALI/ARDS.

Unlike most animal models for ALI that rely on the administration of an insulting agent (toxins, O2, bacteria, etc.), we have developed a pig lung transplantation model that is the reproduction of the lung ischemia/reperfusion injury (IRI) that occurs clinically postsurgical transplantation. A hybrid nanotechnology platform has been created for the sustained expression of anti-inflammatory agents to combat the inflammatory cascade. A "Trojan horse" $[2,3]$ mesoporous silicon (pSi) microparticle is envisioned to escort its nanovector payload to inflammation-associated endothelium, where it is internalized and subsequently releases the therapeutic nanoparticles.[4, 5] Proven silicon fabrication processes allow exquisite control over particle geometry (dimensions from $100 \mathrm{~nm}$ and above) and porosity (pore size range between 5-100 nm) to create particles with specific size, shape, and surface treatments optimized for specific cargo and surface functionalization.[6,7]

Due to their efficient role as gene delivery vectors, a number of viruses are currently being explored for therapeutic gene expression.[8] Because of its lack of pathogenicity, adenoassociated virus (AAV) is one of the most commonly investigated viral vectors. AAV can be used to deliver genes or RNAi [9]. Simple in structure, this virus is composed of a $25 \mathrm{~nm}$ protein capsid harboring a $4.7 \mathrm{kB}$ single-stranded DNA genome. AAV is naturally present in a variety of hosts, including humans, but not pathogenic. To date, at least 12 serotypes have been isolated,[10] with the most commonly used serotype for gene therapy being AAV2. Approximately $80 \%$ of the population has been exposed to this serotype.[11]

We hypothesize that our "Trojan Horse" design concept will provide several important benefits to the immunotherapy approach. First, and the focus of this study, the pSi platform will enable the delivery of large payloads of VNPs into the endothelium, effectively increasing the local dosage of therapeutic genes in the target cells resulting in improved efficacy. Second, the pSi microparticle will shield VNPs from host anti-virus responses, such as neutralization by preexisting antibodies. Furthermore, the microparticle provides a 
facile means of combining multiple types of VNPs, each carrying a different transgene, and ensuring that combinations of genes get delivered to the same target cell. Additionally, other bioactive moieties can be incorporated into the microdevice, further enhancing the immunomodulatory capabilities of the vector. Lastly, the pSi microparticle can be designed to allow for controlled release of VNPs, consequently resulting in the sustained expression of anti-inflammatory factors.

Integrating the use of normothermic ex vivo circulation in conjunction with our novel "Trojan Horse" approach presents an exciting potential technique to effectively reduce the risks associated with systemic delivery by bypassing the negative effects of filter organ clearance and by minimizing the interaction with circulating immune-competent cells. The ex vivo model provides the most promising set-up: an isolated viable organ model with normal metabolic functions. The major drawback of all the present perfusion systems is that their use is based on the concept of preserving the organ at approximately $4^{\circ} \mathrm{C}$ by using a cold flushing solution. The lowering of the cellular metabolic rate is necessary to maintain organ viability. This is an obvious impediment for the delivery of any agents to the allograft, as all the crucial phases depend on metabolic processes. Improved technologies, such as the development of improved pumps and improved ventilators and the development of a lungspecific perfusion solution (Steen solution), which has an optimized composition and oncotic pressure, led to the rebirth of the notion of ex vivo perfusion. The lung has some very peculiar characteristics, as it consists mainly of elastic tissue with a low metabolic requirement. Normally the alveolo-capillary membrane receives its nutrition mostly by direct diffusion and it is possible to sustain the lung tissue by delivering oxygen through the trachea, via mechanical ventilation. Therefore, there is no need to perfuse with a cold solution to lower the metabolic rate. The possibility of treating an entire isolated organ, while circulating a normothermic solution and oxygenating through an endotracheal tube creates the ideal milieu for effectively delivering therapeutic agents ex vivo to address potential complications associated with lung transplant such as IRI.

In this study, we describe optimization of AAV2 loading into pSi microparticles and cell type specific expression of genes delivered by free or encapsulated AAV2. Delivery and expression of the pSi-AAV-GFP vectors ex vivo in vascular endothelium is presented as well as expression of the reporter gene in porcine pulmonary tissue following ex vivo vascular perfusion of the pSi-AAV2-GFP.

\section{Materials and Methods}

\section{Virus production}

AAV2-GFP was produced using HEK293T cells and the triple transfection method.[12] Plasmids pXX6, pXX2, and pAAV-GFP were transfected into cells using polyethyleneimine (PEI). Virus was harvested $72 \mathrm{~h}$ later and purified using density gradient ultracentrifugation. Further purification was performed using a heparin affinity column (GE Healthcare) followed by dialysis into DPBS $(+\mathrm{Mg} / \mathrm{Ca})$. Virus concentration was quantified using QPCR and primers against the CMV promoter contained within the DNA cassette and standards bearing the $\mathrm{CMV}$ promoter. Viral capsids were dissolved with $2 \mathrm{M} \mathrm{NaOH}$ at $56{ }^{\circ} \mathrm{C}$ for 30 mins and then neutralized with $2 \mathrm{M} \mathrm{HCl}$. 


\section{Silicon microparticle functionalization}

Oxidized porous silicon discoidal microparticles with dimensions of $1000 \mathrm{~nm} \times 400 \mathrm{~nm}$ (pore size $\sim 50-60 \mathrm{~nm}$ ) were centrifuged to remove isopropanol and vacuum desiccated. Particles were functionalized with $-\mathrm{NH}_{2}$ (3-aminopropyl-triethoxysilane), $-\mathrm{CH}_{3}$ (methyltrimethoxysilane), -COOH (2-(carbomethoxy) ethyltrimethoxysilane), or -PEI (trimethoxysilylpropyl modified (polyethylenimine)), obtained from Gelest. Dry particles were resuspended in silanes diluted to $2 \%$ in $95 \% \mathrm{v} / \mathrm{v}$ isopropanol in water.

Functionalization occurred for $2 \mathrm{~h}$ at $35^{\circ} \mathrm{C}$ with $1300 \mathrm{RPM}$ shaking. Particles were then rinsed three times with fresh isopropanol and vacuum desiccated in preparation of loading. The surface charge of each particle type was determined by suspending the particles in phosphate buffer and using a Malvern Zetasizer to determine zeta potential.

\section{Loading AAV2-GFP within functionalized particles}

Microparticles were dried (2e8 particles) and then resuspended in AAV2-GFP in DPBS (50 $\mu \mathrm{l}, \sim 1 \mathrm{e} 12$ vector genomes $/ \mathrm{ml})$. Loading was allowed to occur for either 15 or $30 \mathrm{mins}$ at room temperature. After this time particles were pelleted, rinsed twice with sterile water (100 $\mu \mathrm{l}$ each rinse), and then resuspended in $50 \mu \mathrm{l}$ water. Loading was determined using QPCR. The same procedure was used as above, as $2 \mathrm{M} \mathrm{NaOH}$ dissolves silicon microparticles in addition to virus capsids. Presence of dissolved particles was found to not interfere with the PCR reaction (data not shown).

\section{Nanoscale evaluation of pSi surface modification and VNP loading}

Atomic force microscopy (AFM) was used to image surface topography of oxidized or silane-modified (2\% APTES; $2 \mathrm{hr}$ incubation) pSi microparticles. Height and peak force measurements of particles were made using a Bruker Dimension ICON AFM. PeakForce tapping was performed using ScanAsyst to adjust imaging parameters and a model ScanAsyst Air probe made of silicon nitride with a silicon tip, and a spring constant of $0.4 \mathrm{~N} / \mathrm{m}, 1 \mathrm{~Hz}$ scan rate. Analysis of AAV adhesion to a silicon wafer was performed using a Bruker Multimode AFM.

\section{Cell culture}

Gene delivery efficiency was determined in three cell lines: HeLa, HEK293T, and HMVEC (human microvascular endothelial cells). All cells were cultured at $37{ }^{\circ} \mathrm{C}$ with $5 \% \mathrm{CO}_{2}$. HeLa and HEK293T cells were maintained in DMEM with 10\% FBS and 1\% penicillin/ streptomycin. HMVECs were cultured in EGM (Lonza).

\section{Determination of gene delivery using flow cytometry}

Cells were plated in 24 well plates such that they would be $75 \%$ confluent after $24 \mathrm{~h}$. Transduction studies were performed by removing media and replacing with $220 \mu \mathrm{l}$ fresh media. Either free AAV2-GFP or particle-loaded AAV2-GFP (each surface chemistry) were added to the media at a virus MOI of 1,000. After $3 \mathrm{~h}$ the media was removed and replaced with $1 \mathrm{ml}$ fresh media. Cells were then cultured for 3 days before harvesting for flow cytometry analysis of GFP expression. Analysis was performed using a BD Fortessa using the FITC channel to detect GFP expression. 


\section{Confocal imaging of gene expression}

HEK293T cells were seeded in 8 well chamber slides (BD) such that they would be $75 \%$ confluent after $24 \mathrm{~h}$. Media was removed and replaced with $100 \mu \mathrm{l}$. Oxidized, methyl, PEI, or amine functionalized particles loaded with AAV2-GFP were applied such that 30 particles/cell were added in each case. Alternatively, free AAV2-GFP and AAV2-GFP loaded within oxidized pSi microparticles was added at an MOI of 1,000 virus genomes per cell. After $3 \mathrm{~h}$ the media was removed and replaced with $500 \mu \mathrm{l}$ fresh media. Cells were fixed $48 \mathrm{~h}$ later and mounted using VectaShield with DAPI. Control and oxidized particleloaded AAV2-GFP cells were also imaged following labeling with Alexa Fluor® 555 phalloidin (Invitrogen). Slides were imaged using a Nikon A1 confocal microscope.

\section{Pig vein harvesting}

Pigs were put on the operating table in the supine position. General endotracheal anesthesia was administered. A midline sternotomy was performed. The left and right pleural spaces were opened. The pericardium was opened and the heart was exposed. The left pleural reflection was dissected free from the inferior vena cava (IVC), the esophagus and the diaphragm. Then the abdominal cavity was entered just below the pericardial-diaphragmatic attachment, heparin was given and the IVC was cut right at its hepatic junction in order to exanguinate the pig. Using a no-touch technique, the IVC was then cut at its diaphragmatic passage and also at its junction with the right atrium. This allowed for the retrieving of the entire length of the intrathoracic IVC, approximately $4-4.5 \mathrm{~cm}$. The IVC samples were immediately stored in DMEM/F12.

For AFM imaging, the IVC was collected in PBS, fixed overnight in 10\% formalin, and mounted on an AFM magnetic disc, secured with a homemade metal clip, and suspended in PBS. Images were acquired using a MLCT cantilever with a $0.01 \mathrm{nN} / \mathrm{m}$ spring constant in contact mode with the Bruker Multimode

\section{Treatment of pig veins with virus samples}

To load veins with virus, one end was ligated using a 2-0 silk tie. The veins were filled with either media (DMEM/F12 with 5\% BSA and $1 \%$ antibiotic), media with oxidized pSi-AAV (8e8 particles), or media containing an equal number of free viral vectors). The open end of the vein was then ligated using the same technique. Veins were then placed in T25 flasks containing $30 \mathrm{ml}$ media. Samples were placed in a $37^{\circ} \mathrm{C}$ incubator with $5 \% \mathrm{CO}_{2}$ with slow rocking. After a $40 \mathrm{~h}$ incubation, samples were moved to a 6-well plate containing PBS and imaged using an IVIS imaging system.

\section{Particle and tissue imaging using electron microscopy}

For particle imaging, pSi particles, empty or loaded with VNPs, were dried on SEM stubs. SEM images were acquired under high vacuum, at $10-15 \mathrm{kV}$, spot size 3.0 , using a Nova ${ }^{\mathrm{TM}}$ NanoSEM Scanning Electron Microscope (FEI Company, Hillsboro, OR). Gamma levels of the micrographs were adjusted to enhance image contrast and brightness.

For transmission electron microscopy, tissues were fixed in $2 \%$ glutaraldehyde in $0.1 \mathrm{M}$ cacodylate buffer, $\mathrm{pH}$ 7.4. After washing with cacodylate buffer, cells were incubated in a 
mixture of osmium tetroxide and $1 \%$ potassium ferrocyanate in $0.1 \mathrm{M}$ cacodylate buffer for $30 \mathrm{~min}$ at $48^{\circ} \mathrm{C}$. Cells were then dehydrated in increasing concentrations of ethanol and embedded with a mixture of epon and araldite. Ultrathin sections were counter-stained with uranyl acetate and imaged using a JEOL 1210 microscope equipped with an AMT Imaging System. Gamma adjustments were made to the micrographs to enhance image contrast and brightness.

\section{Lung harvest from domestic pig}

Houston Methodist Department of Comparative Medicine (CMP) veterinary staff administered analgesics, induced anesthesia, and maintained animal on anesthesia. The CMP veterinary staff placed the endotracheal tube in the trachea via the laryngeal opening (tube no. 5.5-7). Anesthesia and muscular relaxation was maintained with a inhalant isoflurane at $1-5 \%$. A volume controlled, pressure-regulated ventilation of $10 \mathrm{Llmin}$ ( 20 breaths $/ \mathrm{min}$, positive end-expiratory pressure $=8 \mathrm{~cm} \mathrm{H}_{2} \mathrm{O}$, inspired oxygen fraction $=0.5$ ) was used. The incision site was prepped with 3 alternating washes of chlorohexadine and ethanol followed by placement of topical lidocaine cream. A median sternotomy was performed. The left and right pleural spaces were entered. Heparin $(4 \mathrm{mg} / \mathrm{kg}$ ) was given intravenously. All parts of the lungs were inspected carefully and the positive end-expiratory pressure was increased temporarily to $15 \mathrm{~cm} \mathrm{H}_{2} \mathrm{O}$ for 5 mins so that all atelectasis were eliminated. The inferior and superior vena cavae were isolated and the trachea encircled with a tape. A purse-string suture was placed in the main pulmonary artery. A bolus of 15,000 units of IV heparin (300 units per kilogram) were given; and the pulmonary artery was cannulated with a $21 \mathrm{~F}$ arterial cannula and hooked to the preservation solution tubing, which was flushed of air. We employed $60 \mathrm{~mL} / \mathrm{kg}$ Perfadex and used a $3.3 \mathrm{mmol} / \mathrm{mL}$ of THAM solution per Liter to adjust the $\mathrm{pH}$ to 7.4. The superior and inferior vena cava were ligated as far away from the heart as feasible. After the Perfadex administration was complete, the ascending aorta was cross-clamped, and the trachea was stapled under a positive airway pressure of $20-25 \mathrm{mmHg}$. The rest of the posterior mediastinal attachments were released and the heartlung bloc was explanted. The animal was euthanized via exsanguination when the heart/lung en bloc was removed. On the back table, the heart was excised with careful attention to leave behind an adequate margin of left atrium. The lung bloc was then stored on ice at $4{ }^{\circ} \mathrm{C}$ with a small amount of Perfadex solution in the bag for transport to the laboratory.

\section{Ex-Vivo perfusion of pig lung with AAV2-GFP loaded pSi microparticles}

The left atrial (LA) appendage was trimmed off and the perfusate was allowed to drain freely so that the left atrial pressure was not increased. The solution was collected in a reservoir that feeds the circuit. The pulmonary artery (PA) cannula and an endotracheal tube were attached to the donor lung and connected to the circuit. Flow was initiated slowly in a retrograde fashion to remove air from the circuit. The PA cannula was then connected to the circuit and anterograde flow was started at $150 \mathrm{ml} / \mathrm{min}$ with an acellular perfusate (Steen solution, Vitrolife) at room temperature. The temperature of the perfusate was then gradually increased to $37^{\circ} \mathrm{C}$. When a temperature of $32^{\circ} \mathrm{C}$ to $34^{\circ} \mathrm{C}$ was reached (usually in 30min), ventilation was started and the perfusate flow rate gradually increased. The solution was recirculated for the entire duration of the ex vivo perfusion. The flow of gas used to deoxygenate and add carbon dioxide to the inflow perfusate via a gas exchange 
membrane was then initiated. Mean PA pressures were maintained between 10 and $15 \mathrm{mmHg}$. A protective mode of mechanical ventilation was applied with a tidal volume of $7 \mathrm{ml} / \mathrm{kg}$ at $7 \mathrm{bpm}$, PEEP of $5 \mathrm{cmH} 2 \mathrm{O}$. The lungs were expanded with inspiratory holds to an airway pressure of $20 \mathrm{cmH} 2 \mathrm{O}$ every $\mathrm{hr} . \mathrm{pH}, \mathrm{pCO} 2$, electrolytes, and glucose were maintained at physiologic concentrations in the perfusate. Once equilibrium and physiological conditions were achieved, AAV2-GFP-pSi particles $\left(5 \times 10^{9}\right.$ oxidized $\mathrm{pSi}$ microparticles; $2.6 \mu \mathrm{m}$ diameter hemispherical loaded with $5 \times 10^{11} \mathrm{VNPs}$ ) were introduced via an inlet in the pulmonary artery cannula (perfusing solution) for endothelial uptake. After the ex vivo perfusion/ventilation time of 6 hrs, the lungs were removed from the EVLP system for sample collection.

\section{Lung sample collection and analysis}

After $6 \mathrm{hrs}$ of perfusion with the nanoparticle loaded AAV, samples of the perfusate were collected and $1 \mathrm{~cm}$ cube parenchyma samples were taken from the lower left lobe and lower right lobe of the lungs. From each sample taken, one-fifth was snap frozen in liquid nitrogen, another one-fifth was frozen in mounting medium, another one-fifth was placed in RNA. Subsequently, another one-fifth was placed in $10 \%$ buffered formalin, and the final one-fifth was fixed in $2.5 \%$ gluteraldehyde in sodium cacodylate buffer (Fisher Scientific, Pittsburgh, PA). Lungs were then kept at $\sim 14^{\circ} \mathrm{C}$ overnight and samples were collected from pulmonary artery, pulmonary vein, and lung parenchyma. These samples were distributed in the same way as previously stated. Tissues in mounting medium were sectioned $(8-10 \mu \mathrm{m})$ and fixed in cold acetone for 20 mins. Some sections from each piece were stained for Hematoxylin and Eosin (Fisher Scientific) to identify nuclei and cellular structures using light microscopy. Our particles are visible using light microscopy and therefore we were able to image the particles in relation to the cell nuclei of the tissue samples.

\section{Real-time PCR of GFP expression}

Expression of GFP was determined $11 \mathrm{hrs}$ after perfusion using quantitative real-time PCR. GFP primer sequences were FP 5"-AGC AAA GAC CCC AAC GAG AA-3"; RP 5"-TCG TCC ATG CCG AGA GTG AT- $3^{\prime \prime}$. mRNA was generated using the forward primer and SYBR green was used to detect gene amplification using an annealing temperature of $53^{\circ} \mathrm{C}$. Expression was normalized to $18 \mathrm{~s}$ rRNA using $2^{\wedge}$-deltaCt.

\section{Results}

\section{AAV2 preferentially loads in negatively charged and hydrophobic pSi particles}

A number of silicon surface variations were tested to determine those that were most favorable for virus loading as well as efficient for release and subsequent gene delivery. In addition to oxidized particles, we used silane chemistry to modify the surfaces to be hydrophobic $\left(-\mathrm{CH}_{3}\right)$, positively charged $\left(-\mathrm{NH}_{2},-\mathrm{PEI}\right)$, or negatively charged (-COOH). Zeta potential measurements were performed to determine the final charge of each particle variant (Table 1).

Capillary loading was performed to place AAV2 capsids within the modified $\mathrm{pSi}$ microparticles. Microparticles were loaded for either $15 \mathrm{~min}$ or $30 \mathrm{~min}$, with no significant 
increase in loading shown with a longer incubation time (data not shown). To quantitate the number of particle-associated capsids, both silicon and viral nanoparticles (VNP) were dissolved and QPCR was performed to determine the number of viral genomes per silicon microparticle (Figure 1a). Interestingly, we observe that the more negatively charged surfaces (oxidized and carboxyl) along with the more hydrophobic methyl surface are more efficient for virus localization. With the highest degree of loading, oxidized microparticles carried an average of 1,000 viral capsids per microparticle. Carboxyl and methyl surfaces yielded loading efficiencies of 500 and 600 viral capsids, respectively. The more positively charged amine and PEI modified silicon particles resulted in a minimal level of loading, with an average between 10-50 viral particles per microparticle. When we compare the presence of viral vectors in each loading fraction for oxidized and amine microparticles, we observe that the lack of viral loading within positively charged particles appears to be due to a lack of initial association and not removal upon washing (Figure 1b).

\section{Incorporation of AAV2 within pSi microparticles increases transduction efficiency}

Confocal imaging was used to visualize positive GFP expression in control and AAV-GFP treated 293T cells (Figure 1c). Low levels of GFP expression could be seen in select cells 48 hrs following transduction. Cells were also treated with an equal number of $\mathrm{pSi}$ microparticles (containing viral vectors) using 30 microparticles per cell. After a $3 \mathrm{hr}$ incubation, particles were removed and fresh media was added to the cells. Cells were fixed and imaged $48 \mathrm{hrs}$ post-exposure. Similar to the observations from our flow cytometry studies, the highest levels of GFP expression were observed through the use of negatively charged and hydrophobic particle loading (Figure 1d). Little expression was seen with amine particle loading. Comparing free AAV2-GFP to oxidized pSi-AAV-GFP with an identical MOI of 1,000 demonstrates not only the increase of GFP-positive cells with loaded vectors but also an increase in brightness. This increase in brightness could be attributed to the delivery of multiple AAV2-GFP vectors through the uptake of single pSi microparticles.

\section{Characterization of VNP-loaded pSi microparticles}

An array of pSi microparticles is shown by scanning electron microscopy in Figure 2a.

Higher magnification images reveal the porous structure of unloaded microparticles and the abundant AAV on the surface and within the pores following capillary loading of dry pSi microparticles. Sizes of surface entities on the back face of the pSi microparticle, shown in green, are in line with that expected for VNPs.

Oxidized pSi height and peak force measurements were acquired using a $500 \times 500 \mathrm{~nm}$ grid, with an Rq of $14.4 \mathrm{~nm}, \mathrm{Ra} 11.5 \mathrm{~nm}$ and Rmax $105 \mathrm{~nm}$ (Figure 2b). Roughness measurements for silane modified (APTES) pSi were Rq $13.8 \mathrm{~nm}$, Ra $10.2 \mathrm{~nm}$, and Rmax $101 \mathrm{~nm}$, showing a slightly reduced surface roughness value and peak to valley height, due to the addition of silane to the surface of the $\mathrm{pSi}$ particle. The relatively low reduction in Rmax indicates that the silane coating has not clogged the porous matrix of the pSi particle. The 3D micrograph shows a monolayer of densely packed VNPs on the microparticle surface. The size range of VNPs across the entire scanning field is illustrated in the histogram, with select particle dimensions shown in the bottom graph. The selected VNPs were approximately $15-20 \mathrm{~nm} \times 30-40 \mathrm{~nm}$. 


\section{Cell type specific expression of viral-encoded GFP}

Efficient loading of an abundance of AAV2 capsids within a single pSi microparticle suggests that this delivery mechanism would lead to high levels of localized gene expression. To test this, we applied free AAV2-GFP and pSi-incorporated AAV2-GFP at an equal multiplicity of infection (MOI= 1K) to three cell types. HeLa and 293T cells are highly permissive to AAV2 infection and serve as positive controls. HMVECs, however, are only minimally permissive to AAV2 gene delivery and were chosen to test the hypothesis that incorporation into $\mathrm{pSi}$ would increase transduction, as $\mathrm{pSi}$ microparticles are readily taken up by endothelial cells. Free or loaded vectors were applied for $3 \mathrm{~h}$, removed, and cells were supplemented with fresh media. After $72 \mathrm{~h}$, cells were harvested and the percentage of cells positively expressing GFP was determined using flow cytometry. Expression of HeLa cells following treatment with 5,000 or 10,000 MOI of free VNPs lead to high levels of GFP expression (Figure 3a). Confocal micrographs show high levels of GFP expression in 293T cells (Figure 3b), and as expected, free AAV2-GFP resulted in higher levels of gene expression in HeLa and 293T cells over HMVEC (Figure 1c). Interestingly, for most cases loading with pSi particles maintained or even increased the efficacy of viral performance. In particular, oxidized surfaces generate a 3-5 fold increase in gene expression over AAV2GFP alone, depending on the cell type. Not only do AAV2 vectors favorably associate with pSi microparticles of various chemistries, but loading of large numbers of VNPs into these scaffolds lead to an increase in delivery efficiency.

\section{Successful gene delivery in tissue using virus-loaded microparticles}

Having shown that AAV2 incorporation within oxidized silicon particles leads to increases in gene expression in cultured cells, we sought to test the performance of our platform within a tissue model. To evaluate our technology, we used ex vivo pig vein tissue to compare expression of free AAV2-GFP with that loaded in $\mathrm{pSi}$ microparticles. AAV2-GFP vectors were loaded within oxidized $\mathrm{pSi}$ microparticles $\left(8 \times 10^{8}\right.$ particles $)$ for 30 mins. Excised pig veins were loaded with control media, free AAV-GFP or pSi-AAV-GFP and sutured at both ends. Equal numbers of viral vectors were applied in the latter two cases. Samples were immersed in DMEM/F12 supplemented with 5\% BSA and 1\% antibiotic for $40 \mathrm{hr}$ at $37^{\circ} \mathrm{C}$ with gentle rotation. Veins were imaged for GFP expression using an IVIS imager system (Figure 4a). While free AAV-GFP alone yields GFP gene expression (center), we observe a significant increase in delivery when AAV is loaded within the pSi microparticles (right). The experiment was repeated $4 \mathrm{x}$ with a second example shown in Figure 4b. In the latter experiment, serum was increased to $30 \%$ and gentamicin/ amphotericin B was included in the media. Relative GFP expression is shown in the table in Figure $4 \mathrm{~b}$, with the large ROI encompassing the entire vein segment, and the small ROI limited to the most central region of each section.

\section{Imaging the endothelium and examining endothelial uptake of pSi microparticles}

The AFM height image shows surface topography of the luminal endothelium, with emphasis on the cell body of a single endothelial cell (Figure 5a). Scanning electron micrographs extend across a series of endothelial cells with protrusions representing internalized microparticles (Figure 5b). The boxed region in Figure $5 \mathrm{~b}$ is amplified in the 
adjacent micrograph (Figure 5c) to show that the size and shape of the protrusions are consistent with that of discoidal pSi microparticles. TEM imaging confirms $\mathrm{pSi}$ microparticle uptake by luminal endothelial cells (Figure 5d,e; arrows). Select regions showing microparticles (unloaded or VNP-loaded) are magnified in the lower images.

\section{Ex-Vivo perfusion of pig lung with AAV2-GFP loaded pSi microparticles}

A photograph of the extracted pig lung in the EVLP system is shown in Figure 6a. AAV2pSi microparticles were introduced intravascularly through the cannulated pulmonary artery. The luminal surface of a pulmonary vessel, isolated from the right upper lobe, is presented in the scanning electron micrograph in Figure 6b. Following a $6 \mathrm{hr}$ perfusion of AAV2-pSi microparticles and overnight incubation at $14^{\circ} \mathrm{C}$, tissues were harvested from select regions of pulmonary artery, pulmonary vein, and lung parenchyma. A section of the pulmonary vein stained with $\mathrm{H} \& \mathrm{E}$ is shown in Figure $6 \mathrm{c}$, with particles indicated by arrows. Relative expression of the GFP reporter gene throughout the lung, as determined by RT-PCR, is presented in Figure 6d. While expression was low after the $6 \mathrm{hr}$ perfusion, levels increased in both lung parenchyma and blood vessels after overnight incubation.

\section{Discussion}

We have developed a hybrid particle gene delivery platform based on pSi and AAV. Viral vectors are an optimal gene delivery vehicle for use within this platform, as AAV inherently has mechanisms for endosomal escape and nuclear localization.[13, 14] Following cellular uptake of silicon microparticles, the virus is likely capable of rupturing the endosomal membrane and becoming free within the cytoplasm enabling the genetic cargo to successfully undergo nuclear translocation for transcription. Future studies will focus on uncovering the mechanistic details of pSi-AAV uptake, release from $\mathrm{Si}$, virus intracellular routing, and nuclear translocation in target cells.

Optimal loading of AAV onto pSi microparticles is achieved using oxidized silicon, which presents hydroxyl units. Carboxyl and methyl functionalized pSi surfaces are also able to adsorb large numbers of AAV. Prior work using alkanethiols (displaying various chemical functional groups) patterned onto gold surfaces demonstrated AAV2 can adsorb to many surface chemistries, with the methyl surface yielding highest virus attachment.[15] Differences in outcome between $\mathrm{pSi}$ and alkanethiol-gold surfaces may be due to topographical differences.

Our results indicate that gene delivery from $\mathrm{pSi}$ scaffolds is efficient, and that localization increases the efficiency of gene delivery in some cases. This indicates that not only can AAV be loaded into pSi, but that the AAV is successfully released from the microparticle and is able to deliver genes into the cellular nucleus. We hypothesize this increase in efficacy could be due to a number of reasons. First, pSi microparticle-mediated uptake would override the need for receptor-mediated uptake of AAV into the cell, enabling delivery to non-permissive cells. This process may be more efficient in some cell types. Also, by confining many (up to 1,000) viral particles into one microparticle, the payload of vectors is greatly increased. The occurrence of only one uptake event would be equivalent to many more virus-mediated entries and thus more effective. 
In addition to cultured cells, $\mathrm{pSi}$-AAV vectors demonstrate increased efficacy within ex vivo tissue samples. In particular, using oxidized pSi microparticles, we observed increased gene expression over free virus alone in porcine venous tissue. Efficacy within this model demonstrates that our platform is capable of efficient gene delivery within primary cells in a heterogeneous cellular environment, a point critical for success. Most importantly, we demonstrated that whole organs can be genetically modified using our particle platform following intravenous introduction of the scaffold. Ultimately, we have demonstrated a novel, delivery platform that may in the future be used for sustained delivery of immunemodulators and may be a powerful technique for improving organ or vessel transplant. Future studies will explore avoidance of immunogenicity based on shielded delivery of VPNs within the scaffold.

\section{Acknowledgments}

Plasmids pXX2 and pXX6 were obtained from the Gene Therapy Center at the University of North Carolina. Authors acknowledge the use of The Methodist Hospital Research Institute Scanning Electron and Atomic Force Microscopy, Flow Cytometry, and Advanced Cell and Tissue Microscopy cores. The authors thank Jim Barrish and the Texas Children"s Hospital Electron Microscopy Core for cell and tissue processing and TEM imaging and Heidi Reinhardt, Daniel Hwang, and Rodrigo Diaz for technical assistance. This research was supported by the Alliance for NanoHealth \#W81XWH-11-02-0168.

\section{References}

[1]. Rubenfeld GD, Caldwell E, Peabody E, Weaver J, Martin DP, Neff M, Stern EJ, Hudson LD. Incidence and outcomes of acute lung injury. The New England journal of medicine. 2005; 353:1685-1693. [PubMed: 16236739]

[2]. R.F. Service, Nanotechnology. Nanoparticle Trojan horses gallop from the lab into the clinic. Science. 2010; 330:314-315. [PubMed: 20947742]

[3]. Choi MR, Stanton-Maxey KJ, Stanley JK, Levin CS, Bardhan R, Akin D, Badve S, Sturgis J, Robinson JP, Bashir R, Halas NJ, Clare SE. A cellular Trojan Horse for delivery of therapeutic nanoparticles into tumors. Nano letters. 2007; 7:3759-3765. [PubMed: 17979310]

[4]. Godin B, Tasciotti E, Liu X, Serda RE, Ferrari M. Multistage nanovectors: from concept to novel imaging contrast agents and therapeutics. Accounts of chemical research. 2011; 44:979-989. [PubMed: 21902173]

[5]. Serda RE, Godin B, Blanco E, Chiappini C, Ferrari M. Multi-stage delivery nano-particle systems for therapeutic applications. Biochim Biophys Acta. 2010

[6]. Serda RE, Ferrati S, Godin B, Tasciotti E, Liu X, Ferrari M. Mitotic trafficking of silicon microparticles. Nanoscale. 2009; 1:250-259. [PubMed: 20644846]

[7]. Serda RE, Mack A, Pulikkathara M, Zaske AM, Chiappini C, Fakhoury J, Webb D, Godin B, Conyers JL, Liu XW, Bankson JA, Ferrari M. Cellular Association and Assembly of a Multistage Delivery System. Small. 2010

[8]. Waehler R, Russell SJ, Curiel DT. Engineering targeted viral vectors for gene therapy. Nat Rev Genet. 2007; 8:573-587. [PubMed: 17607305]

[9]. Xia H, Mao Q, Eliason SL, Harper SQ, Martins IH, Orr HT, Paulson HL, Yang L, Kotin RM, Davidson BL. RNAi suppresses polyglutamine-induced neurodegeneration in a model of spinocerebellar ataxia. Nature medicine. 2004; 10:816-820.

[10]. Schmidt M, Voutetakis A, Afione S, Zheng C, Mandikian D, Chiorini JA. Adeno-associated virus type 12 (AAV12): a novel AAV serotype with sialic acid- and heparan sulfate proteoglycan-independent transduction activity. Journal of virology. 2008; 82:1399-1406. [PubMed: 18045941]

[11]. Moskalenko M, Chen L, van Roey M, Donahue BA, Snyder RO, McArthur JG, Patel SD. Epitope mapping of human anti-adeno-associated virus type 2 neutralizing antibodies: 
implications for gene therapy and virus structure. Journal of virology. 2000; 74:1761-1766. [PubMed: 10644347]

[12]. Xiao X, Li J, Samulski RJ. Production of high-titer recombinant adeno-associated virus vectors in the absence of helper adenovirus. Journal of virology. 1998; 72:2224-2232. [PubMed: 9499080]

[13]. Grieger JC, Snowdy S, Samulski RJ. Separate basic region motifs within the adeno-associated virus capsid proteins are essential for infectivity and assembly. Journal of virology. 2006; 80:5199-5210. [PubMed: 16699000]

[14]. Johnson JS, Li C, DiPrimio N, Weinberg MS, McCown TJ, Samulski RJ. Mutagenesis of adenoassociated virus type 2 capsid protein VP1 uncovers new roles for basic amino acids in trafficking and cell-specific transduction. Journal of virology. 84:8888-8902. [PubMed: 20573820]

[15]. McConnell KI, Han A, West JL, Suh J. Microcontact printing for co-patterning cells and viruses for spatially controlled substrate-mediated gene delivery. Soft Matter. 2011; 7:4993-5001. S.J. 


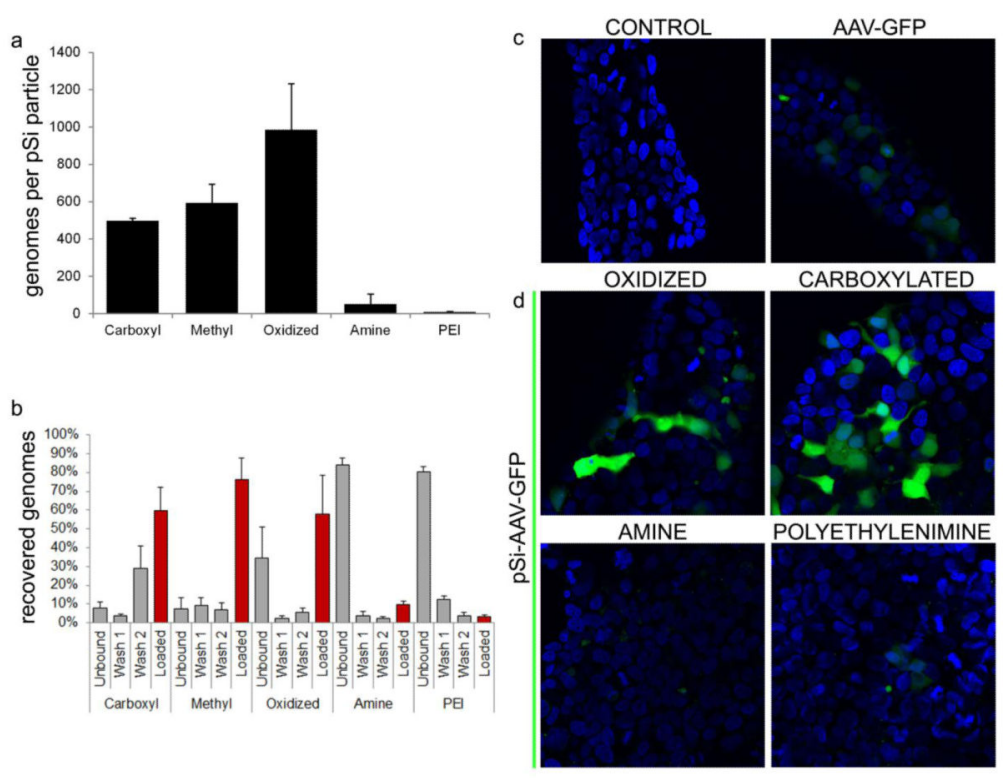

Figure 1. Surface chemistry of pSi microparticles dictates AAV2 loading efficiency and subsequent gene expression

(a) QPCR was used to determine the percent of viral vectors loaded within methyl, carboxyl, amine, oxidized, or PEI functionalized microparticles (red bars), in relation to that remaining unbound and found in each of the two wash steps (gray bars). (b) Absolute number of AAV genomes loaded per pSi microparticle as a function of surface modification. (c) Confocal imaging of control and AAV-GFP $(\mathrm{MOI}=1 \mathrm{~K})$ treated $293 \mathrm{~T}$ cells following a $48 \mathrm{hr}$ incubation and DAPI staining. (d) pSi microparticles with varying surface modifications were loaded with equivalent amounts of AAV-GFP $(\mathrm{MOI}=1 \mathrm{~K})$, followed by 2 wash steps. The hybrid particles were introduced to $293 \mathrm{~T}$ cells for $48 \mathrm{hrs}$ at a dose of $30 \mathrm{AAV}$-loaded pSi microparticles per cell, stained with DAPI and imaged using confocal microscopy. 

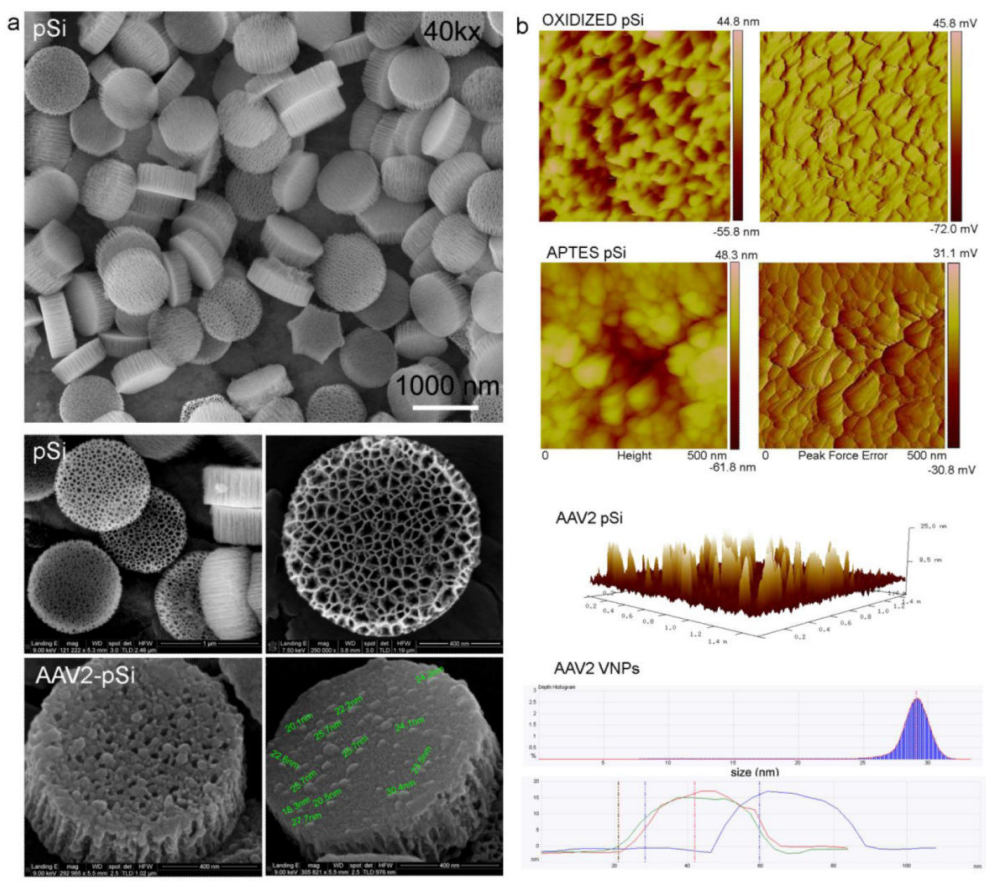

Figure 2. Scanning electron and atomic force microscopy analysis of AAV loading into pSi microparticles

(a) The top SEM image shows a collection of $1000 \times 400 \mathrm{~nm}$ discoidal pSi microparticles (40kx magnification). The center images show unloaded pSi microparticles at approximately $120 \mathrm{kx}$ and $250 \mathrm{kx}$ magnification. The bottom images are front and back views of AAVloaded pSi microparticles imaged at $300 \mathrm{kx}$ magnification. Select measurements of bound VNPs are shown in green. (b) AFM images show height and peak force measurements of oxidized (top) and APTES-modified (middle) pSi microparticles taken with a Bruker Fastscan. AAV bound to the surface of a silicon wafer is shown in the 3D micrograph, illustrating binding density and single layer adhesion. The histograms show the average size of bound AAV vectors and several overlapping single measurements. 

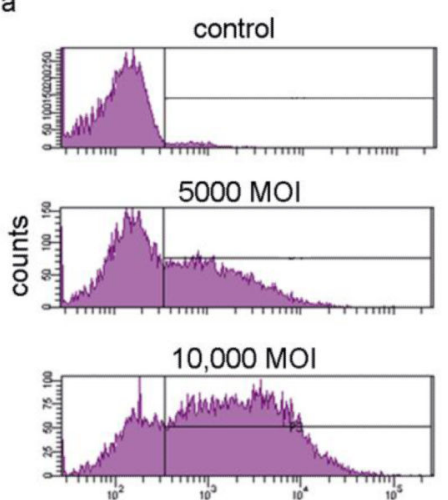

$b$
ํㅡㅁ

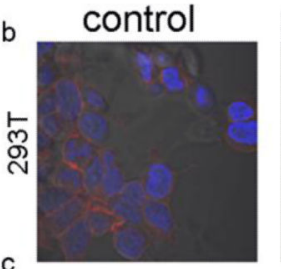

C

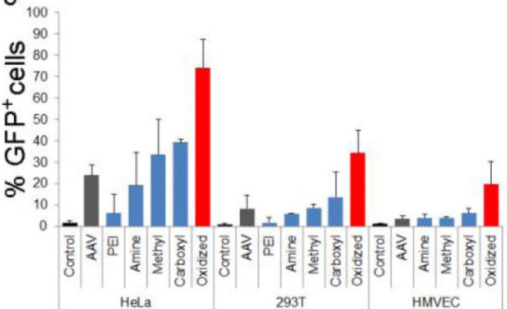

Figure 3. pSi loading and expression of AAV-GFP in a variety of cell types

(a) Flow cytometry histograms show GFP expression in control or AAV (5000 or 10,000 MOI) treated HeLa cells at $72 \mathrm{hrs}$ post treatment initiation. (b) Confocal imaging of 293T cells following $72 \mathrm{hrs}$ incubation with pSi-AAV-GFP. Nuclei are stained with DAPI and the actin cytoskeleton with Alexa Fluor® 555 phalloidin. (c) HeLa, 293T, and HMVEC cells were treated with free AAV-GFP or pSi microparticles of varying surface modification loaded with AAV-GFP at an MOI of 1,000. Flow cytometry was used to detect GFP expression 72 hrs post-delivery. 

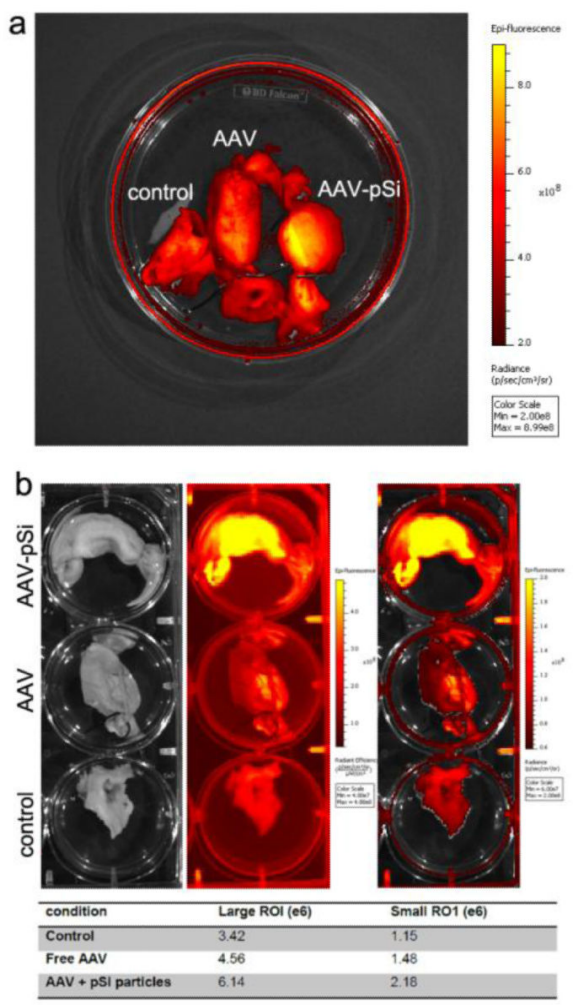

Figure 4. Enhanced gene delivery in pig vein tissue using $\mathbf{p S i}$

(a) Procine inferior vena cava was excised and loaded with media containing either free AAV2-GFP (center), pSi-AAV2-GFP (oxidized; top), or control (bottom). IVIS imaging of tissues after $72 \mathrm{hrs}$ incubation at $37^{\circ} \mathrm{C}$. Fluorescent intensity of ROIs of are shown for each sample. Large ROI reflect the entire loaded region of the vein, while small ROI is the central part of the vein only. (b) Duplicate experiment showing IVIS imaging of the three vein segments treated with media (left), free AAV2-GFP (center), or pSi-AAV2-GFP (right) for 72 hrs. 


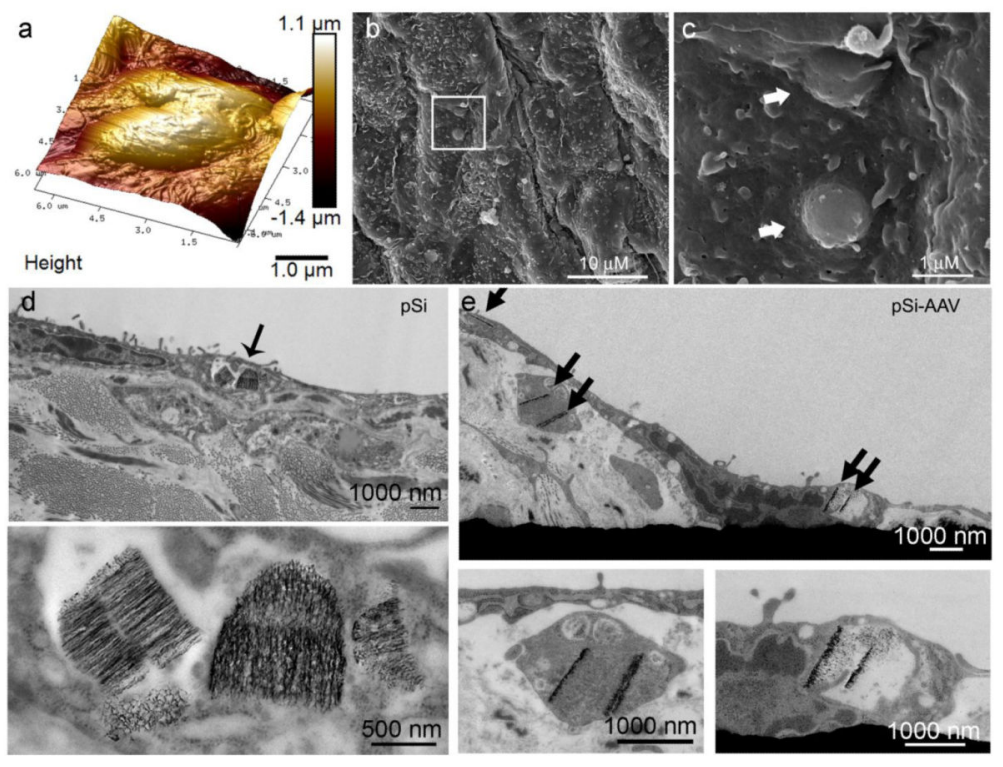

Figure 5. Endothelial uptake of control or AAV2-GFP loaded pSi microparticles (a) AFM image showing surface topography of an untreated section of luminal endothelium of the inferior vena cava. (b,c) Scanning electron micrographs show surface topography of the luminal endothelium following $72 \mathrm{hr}$ incubation with pSi-AAV2. The boxed region in „, $\mathrm{b}^{\prime \prime}$ is amplified in , $\mathrm{c}^{c e}$ to highlight microparticles beneath the endothelial cell membrane (white arrows). (d,e) Transmission electron micrographs of vein specimen at $72 \mathrm{hrs}$ after introduction of control (d) or AAV2-GFP loaded (e) pSi microparticles. Black arrows indicate the location of internalized microparticles, with these regions magnified in the lower images. 


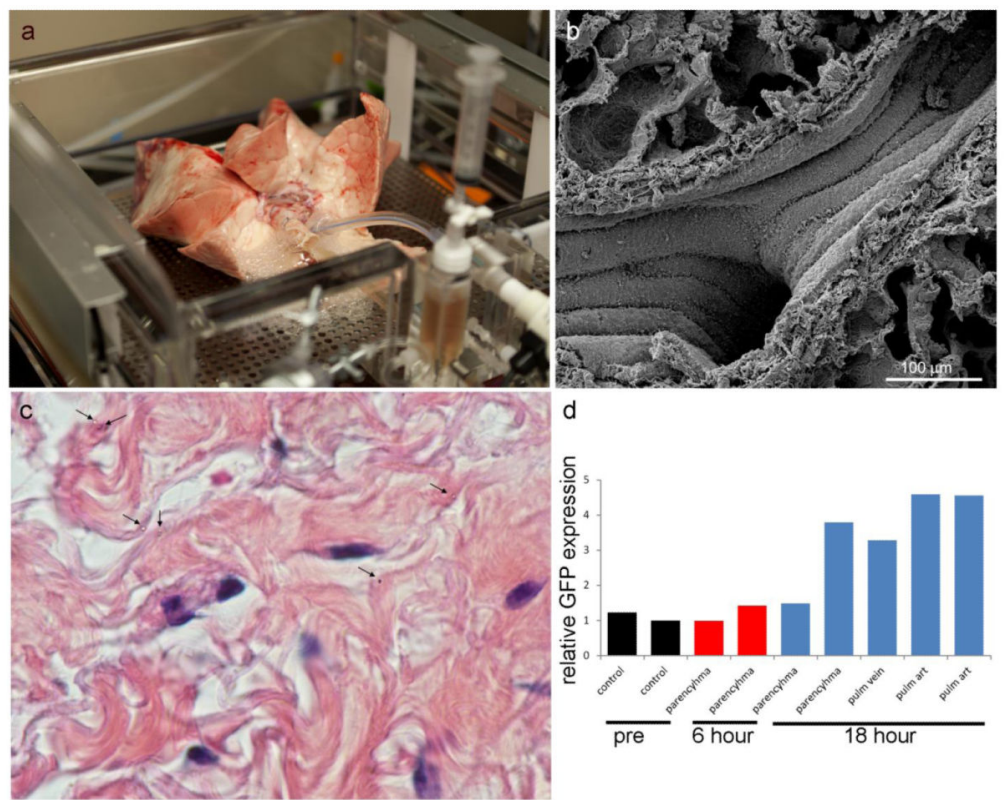

Figure 6. Perfusion of a pig lung with an AAV2-GFP-pSi reporter construct (a) Domestic pig lung on Harvard Apparatus Ex-Vivo Organ Perfusion System. (b) SEM image of a porcine lung blood vessel emphasizing the architecture of the luminal endothelium. (c) Lung pulmonary vein stained with H \& E (40x Magnification). Arrows indicate location of particles. (d) GFP expression in select sections of lung tissue 6 and 18 hrs after vascular perfusion with AAV-GFP-pSi reporter constructs. 


\section{Table 1}

Zeta potential measurements of chemically-modified and viral-loaded pSi scaffolds

\begin{tabular}{ccc} 
Sample & Zeta potential $(\mathbf{m V})$ & Stdev \\
\hline COOH & -20.7 & 8.2 \\
NH2 & 5.6 & 6.1 \\
OH & -35.6 & 5.9 \\
PEI & -1.45 & 6.1 \\
Methyl & -17.0 & 7.5 \\
Oxidized-loaded with AAV2 & -25.1 & 6.5 \\
\hline
\end{tabular}

